\title{
Noninvasive Fetal Electrocardiography in the Diagnosis of Long QT Syndrome: A Case Series
}

\author{
Neeta Sethi ${ }^{a}$ Kiyoe Funamoto ${ }^{b}$ Catherine Ingbar ${ }^{a}$ Paige Mass ${ }^{c}$ \\ Jeffrey Moak ${ }^{a}$ Ronald Wakai ${ }^{d}$ Janette Strasburger ${ }^{\mathrm{e}}$ Mary Donofrio ${ }^{\mathrm{a}}$
}

Ahsan Khandoker ${ }^{f}$ Yoshitaka Kimura $^{b}$ Anita Krishnan ${ }^{a}$

a Division of Cardiology, Children's National Hospital, Washington, DC, USA; ${ }^{b}$ Department of Advanced Interdisciplinary Biomedical Engineering, Tohoku University School of Medicine, Sendai-shi, Miyagi, Japan; ' $S$ heikh Zayed Institute for Pediatric Surgical Innovation, Children's National Hospital, Washington, DC, USA; ${ }^{\text {Biomagnetism }}$ Laboratory, University of Wisconsin-Madison, Madison, WI, USA; ${ }^{e}$ Division of Cardiology, Herma Heart Institute, Children's Hospital of Wisconsin, Milwaukee, WI, USA; ${ }^{f}$ Department of Biomedical Engineering, Khalifa University of Science and Technology, Abu Dhabi, United Arab Emirates

\section{Established Facts}

- Early detection and monitoring for malignant arrhythmias is fundamental to prenatal care in congenital long QT syndrome (LQTS) to prevent perinatal mortality.

- Fetal magnetocardiography (fMCG) is the gold standard to precisely characterize fetal heart rhythm and conduction, but has limitations.

- Historically, fetal electrocardiography (fECG) has been limited by technical challenges in extracting the low-amplitude fetal ECG signal from maternal noise.

\section{Novel Insights}

- A novel signal processing technique of blind source separation has shown improved success in isolating the fetal ECG and measuring cardiac intervals.

- This case series highlights the application of a noninvasive fECG device with blind source separation (IRIS ${ }^{\mathrm{TM}}$; Atom Medical Company, Tokyo, Japan).

- In 3 cases of clinically suspected LQTS, fECG had high diagnostic accuracy compared to current goldstandard tests of fMCG and postnatal testing.

- More research on fECG in fetal arrhythmia and conduction disease is needed, but early results are promising.

\section{Keywords}

Congenital long QT syndrome · Prenatal diagnosis ·

Noninvasive fetal electrocardiography · Arrhythmias

\begin{abstract}
Introduction: Early detection and monitoring for malignant arrhythmias is fundamental to prenatal care in long QT syndrome (LQTS). Recently, we studied the feasibility of isolating the fetal electrocardiogram (fECG) and measuring elec-
\end{abstract}


trocardiographic intervals with a noninvasive fECG device using blind source separation with reference signal. Our aim was to evaluate the ability of fECG to diagnose LQTS. Case Presentations: We identified 3 cases of clinically suspected LQTS based on fetal echocardiogram (2 had sinus bradycardia, 1 had second-degree atrioventricular block with negative maternal anti-SSA/SSB antibody titers). With institutional review board approval, these patients were prospectively enrolled for $\mathrm{fECG}$ acquisition. Offline post-processing generated fECG waveforms and calculated QT intervals. Case 1 and 3 had a maternal history of LQTS. Two of the three fetuses with suspected LQTS had confirmed LQTS by postnatal ECG and genetic testing. FECG was able to identify a prolonged corrected QT interval in both cases. One of these also had fetal magnetocardiography (fMCG), which yielded similar findings to the fECG. The third fetus had a normal fECG; fMCG and postnatal ECG were also normal. Conclusions: In 3 cases, fECG findings corroborated the diagnosis of LQTS. Noninvasive fECG may offer a novel method for fECG that is portable and more clinically accessible.

(c) 2020 S. Karger AG, Basel

\section{Introduction}

Prenatal diagnosis of congenital long QT syndrome (LQTS) with close fetal monitoring is essential to the management of susceptible fetuses in utero and during postnatal transition. Early detection enables preventive measures such as correcting maternal hypomagnesemia or hypocalcemia, as well as avoiding maternal QT-prolonging medications that could reduce the risk of torsades de pointes developing in the LQTS fetus [1]. If malignant ventricular arrhythmias such as ventricular tachycardia (VT) or torsades de pointes are detected, transplacental treatment is recommended and highly successful [2-5]. Additionally, specialized delivery room care can be planned for [6], and primary prevention can be initiated immediately with beta-blockade in the neonate [7].

Prenatal diagnosis of LQTS using conventional antepartum testing is challenging, since fetal electrocardiographic tracings are not conventionally available. In addition, the secondary finding of fetal sinus bradycardia or decreased heart rate variability on cardiotocogram is often subtle and may not be recognized as abnormal by routine obstetrical standards [8].

Fetal echocardiography is the conventional tool for evaluating the mechanism of an abnormal fetal heart rhythm but is limited by its assessment of atrial and ventricular mechanical systoles rather than the cardiac elec- trical waveform $[1,9]$. It cannot assess the electrical waveform morphology or repolarization characteristics. Fetal magnetocardiography (fMCG) is the current gold standard to precisely characterize fetal heart rhythm and conduction, and has been well described in the diagnosis and management of congenital LQTS [10-12]. However, its need for expensive and highly specialized equipment has geographically limited its clinical application $[1,13]$.

The clinical application of fetal electrocardiography (fECG) has been historically limited by technical challenges in extracting the low-amplitude fetal ECG signal from maternal noise [14]. A novel signal processing technique of blind source separation has shown improved success in isolating fetal ECG activity and measuring cardiac electrical intervals [15-17].

This case series highlights the use of a noninvasive 12lead fetal ECG device with blind source separation with reference technique (IRIS ${ }^{\mathrm{TM}}$; Atom Medical Company, Tokyo, Japan) in the diagnosis and management of fetal LQTS. This case series is a part of an institutional review board-approved research protocol at our institution with this device from which early work has been published [18]. Table 1 summarizes the results of all 3 cases. A threshold of a corrected QT (QTc) interval of $\geq 490 \mathrm{~ms}$ by fMCG has been shown to correlate with postnatal genetically confirmed LQTS [11].

\section{Case Presentations}

Case 1

A 21-year-old woman with familial LQTS (KCNE1 and probable $\mathrm{KCNH} 2$ mutations) with a transvenous implantable cardioverter defibrillator, status post left thoracic sympathectomy, and on maintenance of beta-blockade was referred for a screening fetal echocardiogram at 23 weeks' gestation. Fetal echocardiogram revealed a structurally normal heart. Simultaneous pulsed Doppler interrogation of the mitral valve and left ventricular outflow tract revealed normal sinus rhythm with a lower heart rate of $120 \mathrm{bpm}$. The isovolumetric relaxation time was mildly prolonged at $50 \mathrm{~ms}$, which coupled with the lower heart rate and was suggestive of LQTS in the fetus $[19,20]$.

Fetal ECG was performed at that appointment and demonstrated normal sinus rhythm with a prolonged QTc interval of 526 $\mathrm{ms}$ and $\mathrm{T}$ wave flattening, indicative of fetal LQTS. Notably, fECG was able to isolate the fetal signal with a prolonged QT interval from the maternal tracing with LQTS (Fig. 1). The mother was referred for fMCG done at 25 weeks' gestation, which showed an averaged QTc of $573 \mathrm{~ms}$, consistent with LQTS. Fetal ECG was repeated at 27 weeks' gestation with similar findings to the prior fECG and the fMCG (Fig. 2).

Maternal serum calcium and magnesium levels were monitored regularly. Repeat fetal echocardiogram at 27 weeks' gestation was unchanged. She delivered a full-term baby at an outside institution without complications. The baby was admitted to the spe- 


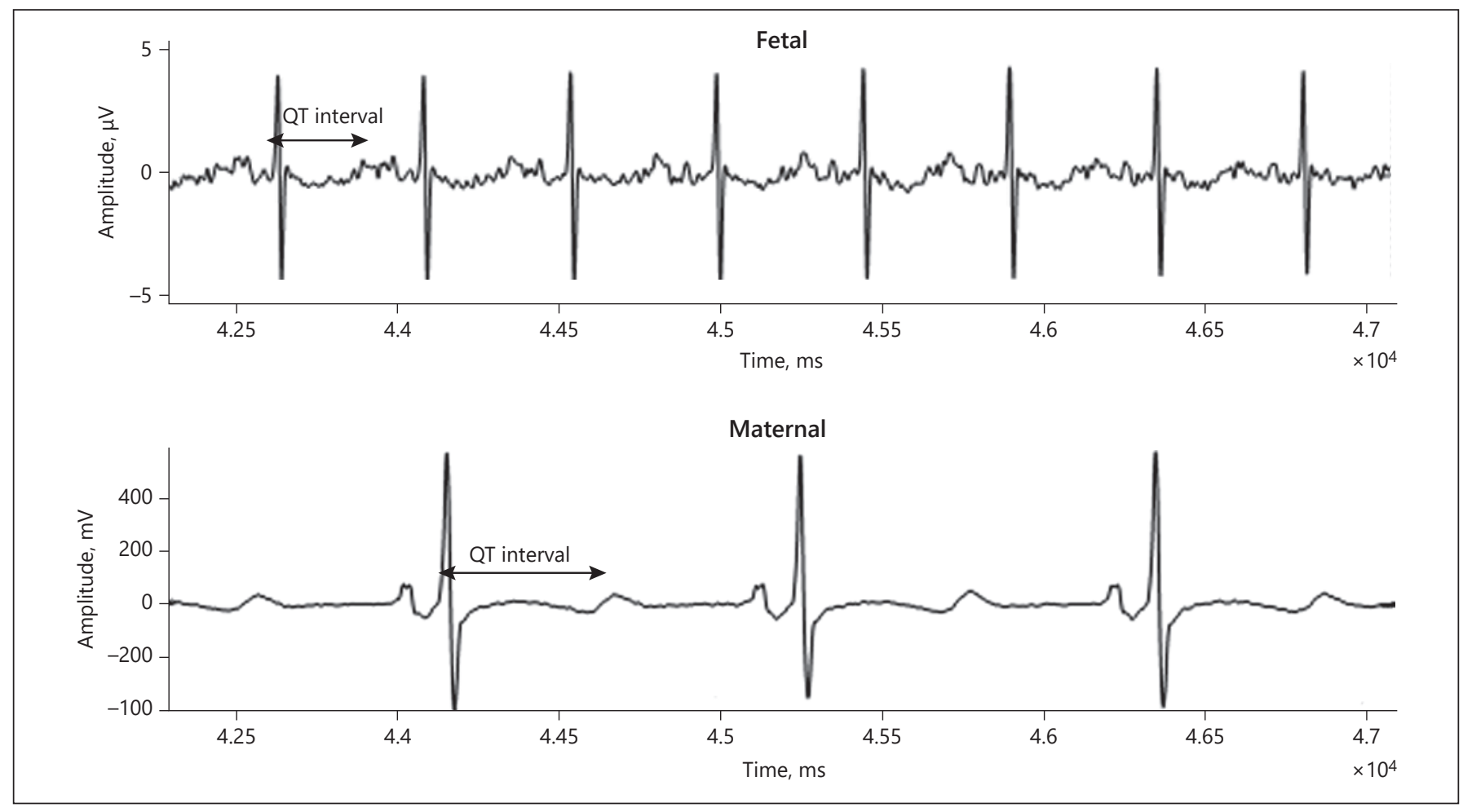

Fig. 1. Case 1. fECG done at 23 weeks' gestation showing prolonged QT interval, isolated from the maternal tracing with LQTS.

Table 1. Summary of results

\begin{tabular}{llllll}
\hline Case & Maternal LQTS & fECG (GA) & fMCG (GA) & Postnatal ECG & $\begin{array}{l}\text { Postnatal genetic test- } \\
\text { ing }\end{array}$ \\
\hline 1 & $\begin{array}{l}\text { Yes, } \text { KCNE1 and } \\
\text { KCNH2 mutations }\end{array}$ & $\begin{array}{l}\text { QTc 526ms (23) } \\
\text { Repeat QTc 544 ms (27) }\end{array}$ & QTc 573 ms (25) & $\begin{array}{l}\text { Positive, QTc } \\
480-572 \mathrm{~ms}\end{array}$ & $\begin{array}{l}\text { Variant KCNH2 gene } \\
\text { (LQTS type2) }\end{array}$ \\
\hline 2 & No & QTc 504 ms (24) & Not done & $\begin{array}{l}\text { Positive, QTc } \\
\text { 484-527 ms }\end{array}$ & $\begin{array}{l}\text { Variant KCNQ1 gene } \\
\text { (LQTS type 1) }\end{array}$ \\
\hline 3 & Yes, gene negative & QTc 433 ms (27) & QTc 451 ms (22) & Normal & Not done \\
\hline
\end{tabular}

LQTS, long QT syndrome; fECG, fetal electrocardiogram; GA, gestational age; fMCG, fetal magnetocardiography; ms, milliseconds.

cial care nursery with a QTc of 480-572 ms and biphasic T-waves on postnatal ECG. The baby had no bradycardia or ventricular arrhythmia, and was discharged home with propranolol. Genetic testing returned positive for a variant in the $\mathrm{KCNH} 2$ gene, confirming the diagnosis of LQTS type 2.

\section{Case 2}

A 37-year-old woman was referred for fetal echocardiogram at 20 weeks' gestation after a routine obstetrical ultrasound that revealed fetal bradycardia. Fetal echocardiogram demonstrated a structurally normal heart. Pulsed Doppler interrogation of rhythm revealed a heart rate of $60 \mathrm{bpm}$ with second-degree atrioventricular heart block. Given the possibility of autoimmune-mediated heart block, transplacental treatment with dexamethasone was started and subsequently discontinued once maternal anti-SSA/ SSB antibody titers returned negative.

Repeat cardiac evaluations demonstrated persistent fetal sinus bradycardia, raising concern for fetal LQTS. Fetal cardiac function remained normal, and there were no signs of fetal hydrops. Fetal ECG at 24 weeks' gestation demonstrated sinus bradycardia with an averaged heart rate of $86 \mathrm{bpm}$ and prolonged QTc of $504 \mathrm{~ms}$. An fMCG was unable to be done.

With prenatal electrophysiology consultation, the mother delivered a full-term baby without complications. In the delivery room, the baby's heart rate was $140 \mathrm{bpm}$, and no resuscitation was required. Postnatal ECG demonstrated QTc prolongation of 484- 


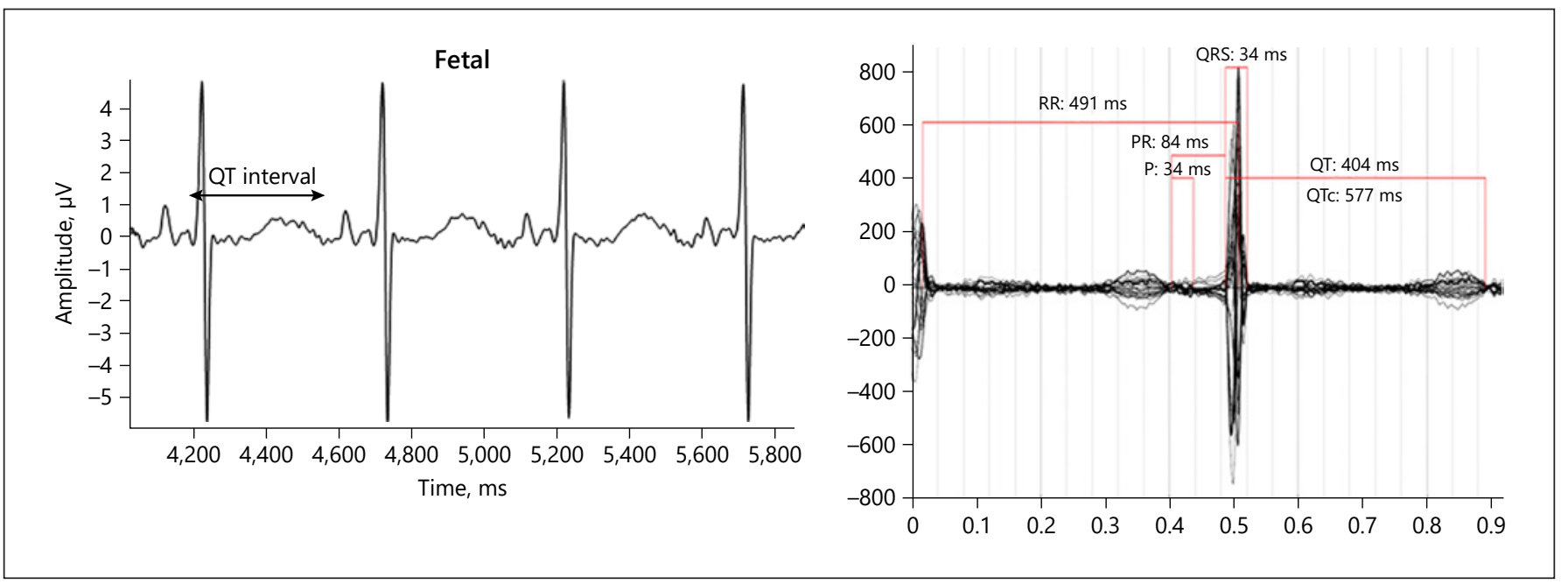

Fig. 2. Case 2. The panel on the left is fECG tracing done at 27 weeks' gestation, demonstrating a low fetal heart rate at $125 \mathrm{bpm}$ and prolonged QTc $544 \mathrm{~ms}$. The panel on the right shows fMCG done at 25 weeks' gestation, also revealing a prolonged QT interval with averaged QTc $573 \mathrm{~ms}$. Both are consistent with LQTS. RR, time between consecutive $\mathrm{R}$ waves, representative of the heart rate; $\mathrm{PR}$, interval between the $\mathrm{P}$ wave and $\mathrm{R}$ wave, reflecting conduction from the atria through the atrioventricular node; P, P wave, representing atrial contraction; QRS, ventricular depolarization.

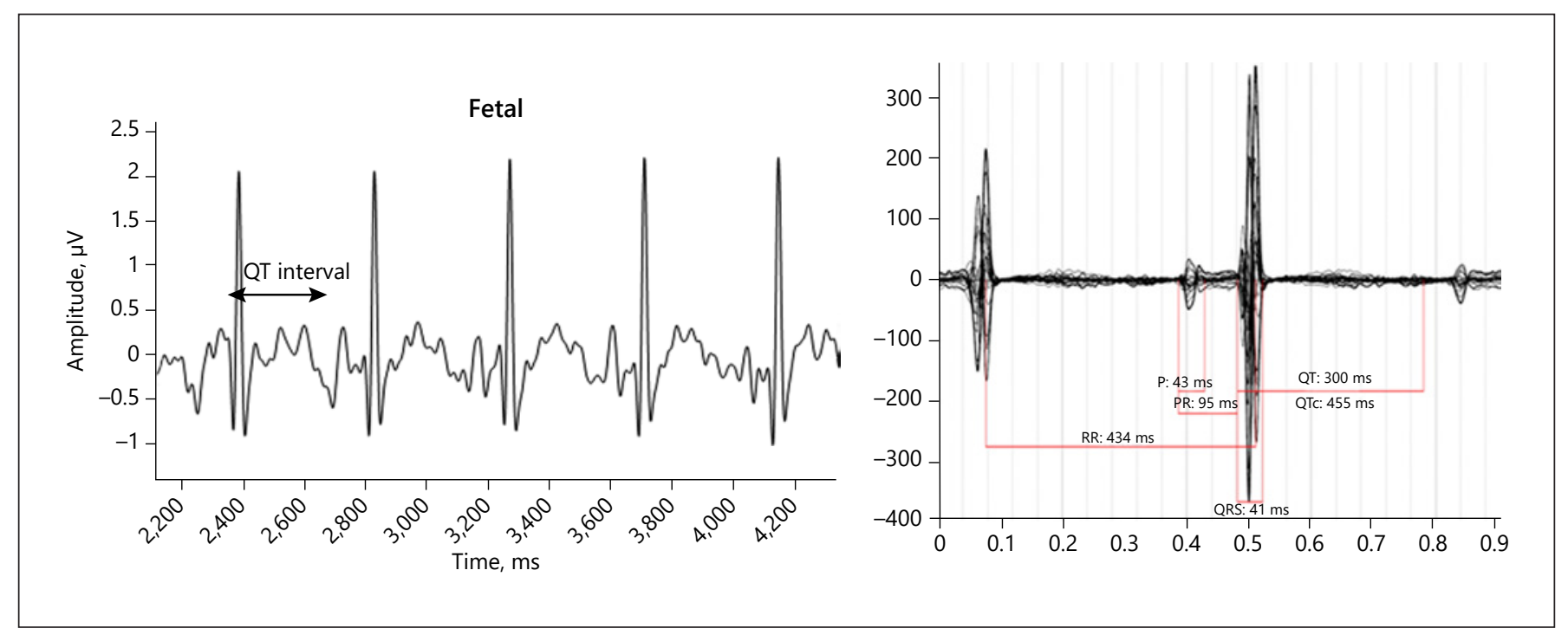

Fig. 3. Case 3. The panel on the left shows fECG done at 27 weeks' gestation, revealing a normal QT interval (QTc $433 \mathrm{~ms}$ ). The panel on the right is fMCG at 22 weeks' gestation; also normal with an averaged QTc $451 \mathrm{~ms}$. RR, time between consecutive R waves, representative of the heart rate; $\mathrm{PR}$, interval between the $\mathrm{P}$ wave and $\mathrm{R}$ wave, reflecting conduction from the atria through the atrioventricular node; P, P wave, representing atrial contraction; QRS, ventricular depolarization.

$527 \mathrm{~ms}$ with T-wave abnormalities. After transfer to our institution, the baby was started on propranolol. Genetic testing revealed a pathologic variant in KCNQ1, confirming the diagnosis of LQTS type 1 . Notably, parental ECGs were normal, and so this likely represented a de novo mutation.
Case 3

A 26-year-old woman with gene-negative LQTS with a transvenous implantable cardioverter defibrillator and maintenance beta blockade was referred for a screening fetal echocardiogram at 20 weeks gestation. Fetal echocardiogram demonstrated a struc- 
turally normal heart. Pulsed Doppler rhythm interrogation demonstrated normal sinus rhythm. Repeat cardiac evaluations revealed no change in fetal or cardiac status, but the heart rate was lower at $115 \mathrm{bpm}$ in the third trimester.

Fetal ECG at 27 weeks found normal sinus rhythm at a rate of $143 \mathrm{bpm}$ with normal QT interval of $433 \mathrm{~ms}$. The mother was referred for fMCG at 22 weeks, which demonstrated a normal fetal heart rate and normal averaged QTc interval of $451 \mathrm{~ms}$; i.e., without evidence of LQTS, as seen on fetal ECG (Fig. 3).

She delivered a full-term baby without complications. The baby was seen at the outpatient cardiology clinic at 1 and 2 months of age with no clinical concerns. ECGs done at those appointments raised no concerns for congenital LQTS with normal QTc intervals and normal T-wave morphology.

\section{Discussion}

This case series is the first to feature the application of fetal ECG using blind source separation in the diagnosis of fetal LQTS compared to the current gold standard tests of fMCG or postnatal testing. In the 2 cases of confirmed LQTS on postnatal ECG and genetic testing, fECG was able to identify a prolonged QT interval in both cases. Significantly, one of these cases had maternal LQTS, and fECG was able to isolate the fetal signal with prolonged QT from the maternal tracing with LQTS. That case also had an $\mathrm{MCG}$ done whose findings suggestive of LQTS were similar to those seen on fECG. The third case of clinically suspected LQTS based on fetal echocardiogram had a normal QT interval on both fECG and fMCG with normal postnatal ECG testing, reinforcing the known limitations of echocardiography to predict electrocardiographic abnormalities. Thus, in our series of three cases, fECG had a high diagnostic accuracy for LQTS compared to conventional gold standard testing.

Blind source separation with reference technique may offer a novel, noninvasive method for fECG that is portable and able to be acquired in a standard patient examination room and thus, more clinically accessible [18]. This is especially valuable in suspected congenital LQTS in which frequent evaluation of the fetal heart rate and rhythm is essential. Furthermore, fetuses at risk for or with clinically suspected LQTS should perhaps be screened more than once, since literature on fMCG in the diagnosis of LQTS has shown that a prolonged QT interval may not be evident until later in gestation on repeat testing [11]. Additionally, repeated evaluations in LQTS-suspected fetuses are needed to monitor for malignant arrhythmias such as long QTinduced atrioventricular block and ventricular tachy- cardia [1]. Frequent screening and monitoring are not as feasible by fMCG in which practice locations are limited. In addition, the acquisition time of fECG being only 10 min makes it easy to integrate into a busy clinical practice. Therefore, the high clinical accessibility of fECG has the potential to significantly alter the clinical care of LQTS-susceptible fetuses.

At this time, there is limited data on the clinical application of fECG, and further advancements in the technology are needed for clearer fECG signal acquisition that can more precisely filter out maternal noise and baseline wandering. Furthermore, at this time, fECG post-processing and measurements of cardiac time intervals are made as part of clinical research by a specialized team and not widely available for independent analysis. Early results of fECG including this case series are promising; however, more data are needed to understand its diagnostic accuracy in fetal arrhythmias and conduction system disease.

\section{Statement of Ethics}

All procedures performed in studies involving human participants were in accordance with the ethical standards of the institutional review board on human research at Children's National Hospital and with the 1964 World Medical Association Declaration of Helsinki and its later amendments or comparable ethical standards. Written informed consent was obtained from all individual participants included in the study.

\section{Disclosure Statement}

The authors have no conflicts of interest to declare.

\section{Funding Sources}

Dr. Krishnan received funding for this work from the Seelig Cardiac Research Fund (Children's National Hospital). The work of Dr. Ronald Wakai with fetal magnetocardiography is supported by NIH Grant RO1HL063174. In this case series, 2 cases underwent fetal magnetocardiography.

\section{Author Contributions}

All authors have participated sufficiently in the research, have approved the manuscript contents, and agree with its submission to the journal of Fetal Diagnosis and Therapy. 


\section{References}

1 Donofrio MT, Moon-Grady AJ, Hornberger LK, Copel JA, Sklansky MS, Abuhamad A, et al.; American Heart Association Adults With Congenital Heart Disease Joint Committee of the Council on Cardiovascular Disease in the Young and Council on Clinical Cardiology, Council on Cardiovascular Surgery and Anesthesia, and Council on Cardiovascular and Stroke Nursing. Diagnosis and treatment of fetal cardiac disease: a scientific statement from the American Heart Association. Circulation. 2014 May;129(21):2183-242.

2 Miyake A, Sakaguchi H, Miyazaki A, Miyoshi T, Aiba T, Shiraishi I. Successful prenatal management of ventricular tachycardia and second-degree atrioventricular block in fetal long QT syndrome. HeartRhythm Case Rep. 2016 Sep;3(1):53-7.

3 Chang IK, Shyu MK, Lee CN, Kau ML, Ko $\mathrm{YH}$, Chow SN, et al. Prenatal diagnosis and treatment of fetal long QT syndrome: a case report [Internet]. Prenat Diagn. 2002 Dec; 22(13):1209-12. Available from: http://doi. wiley.com/10.1002/pd.475.

4 Simpson JM, Maxwell D, Rosenthal E, Gill H. Fetal ventricular tachycardia secondary to long QT syndrome treated with maternal intravenous magnesium: case report and review of the literature. Ultrasound Obstet Gynecol. 2009 Oct;34(4):475-80.

5 Cuneo BF, Ovadia M, Strasburger JF, Zhao H, Petropulos T, Schneider J, et al. Prenatal diagnosis and in utero treatment of torsades de pointes associated with congenital long QT syndrome. Am J Cardiol. 2003 Jun;91(11):1395-8.

6 Greene EA, Berul CI, Donofrio MT. Prenatal diagnosis of long QT syndrome: implications for delivery room and neonatal management. Cardiol Young. 2013 Feb;23(1):141-5.
7 Cuneo BF, Strasburger JF, Wakai RT. The natural history of fetal long QT syndrome [Internet]. J Electrocardiol. 2016 Nov - Dec; 49(6):807-13

8 Ishikawa S, Yamada T, Kuwata T, Morikawa M, Yamada T, Matsubara S, et al. Fetal presentation of long QT syndrome-evaluation of prenatal risk factors: a systematic review. Fetal Diagn Ther. 2013;33(1):1-7.

9 Strasburger JF, Cheulkar B, Wakai RT. Magnetocardiography for fetal arrhythmias. Heart Rhythm. 2008 Jul;5(7):1073-6.

10 Menéndez T, Achenbach S, Beinder E, Hofbeck M, Schmid O, Singer H, et al. Prenatal diagnosis of QT prolongation by magnetocardiography. Pacing Clin Electrophysiol. 2000 Aug;23(8):1305-7.

11 Cuneo BF, Strasburger JF, Yu S, Horigome H, Hosono T, Kandori A, et al. In utero diagnosis of long QT syndrome by magnetocardiography. Circulation. 2013 Nov;128(20):2183-91.

12 Hosono T, Kawamata K, Chiba Y, Kandori A, Tsukada K. Prenatal diagnosis of long QT syndrome using magnetocardiography: a case report and review of the literature [Internet]. Prenat Diagn. 2002 Mar;22(3): 198-200. Available from: http://www.ncbi.nlm.nih. gov/pubmed/11920893

13 Wacker-Gussmann A, Strasburger JF, Cuneo BF, Wakai RT. NIH Public Access. 2015; 31(7):617-28

14 Kimura Y, Sato N, Sugawara J, Velayo C, Hoshiai T, Ito T, et al. Recent Advances in Fetal Electrocardiography [Internet]. Open Med Devices J. 2012 May;4(1):7-12. Available from: http://benthamopen.com/ABSTRACT/ TOMDJ-4-7
15 Agostinelli A, Grillo M, Biagini A, Giuliani C, Burattini L, Fioretti S, et al. Noninvasive fetal electrocardiography: an overview of the signal electrophysiological meaning, recording procedures, and processing techniques [Internet]. Ann Noninvasive Electrocardiol. 2015 Jul;20(4):303-13. Available from: http://doi. wiley.com/10.1111/anec.12259

16 Sato M, Kimura Y, Chida S, Ito T, Katayama $\mathrm{N}$, Okamura K, et al. A novel extraction method of fetal electrocardiogram from the composite abdominal signal. IEEE Trans Biomed Eng. 2007 Jan;54(1):49-58.

17 Sato N, Hoshiai T, Ito T, Owada K, Chisaka $\mathrm{H}$, Aoyagi A, et al. Successful detection of the fetal electrocardiogram waveform changes during various states of singletons [Internet]. Tohoku J Exp Med. 2011 Oct;225(2):89-94. Available from: http://joi.jlc.jst.go.jp/JST. JSTAGE/tjem/225.89? from $=$ CrossRef

18 Doshi AN, Mass P, Cleary KR, Moak JP, Funamoto K, Kimura Y, et al. Feasibility of Noninvasive Fetal Electrocardiographic Interval Measurement in the Outpatient Clinical Setting [Internet]. Pediatr Cardiol. 2019 Aug; 40(6):1175-82.

19 Clur SAB, Vink AS, Etheridge SP, Robles De Medina PG, Rydberg A, Ackerman MJ, et al. Left Ventricular Isovolumetric Relaxation Time Is Prolonged in Fetal Long-QT Syndrome. Circ Arrhythmia Electrophysiol. 2018 Apr;11(4):e005797.

20 Cuneo B, Robles de Medina P, Blom N, Wilde AA, Donofrio MT, Benson DW, et al. 247: Isovolumic relaxation time is prolonged in fetal long QT syndrome [Internet]. Am J Obstet Gynecol. 2017 Jan;216(1):S154. Available from: https://linkinghub.elsevier.com/retrieve/ pii/S0002937816311310. 